\title{
Эффекты локального фотовозбуждения носителей заряда высокой плотности в кремнии
}

\author{
(C) A.M. Мусаев \\ Институт фризики им. Х.И. Амирханова Дагестанского научного центра Российской академии наук, \\ 367003 Махачкала, Россия \\ E-mail: akhmed-musaev@yandex.ru
}

(Получена 17 января 2017 г. Принята к печати 6 февраля 2017 г.)

Экспериментально исследованы явления при локальном импульсном фотовозбуждении собственных, неравновесных носителей заряда высокой концентрации в кремнии. Обнаружен эффект существенного роста времени жизни фотовозбужденных носителей заряда. Показано, что эффект существенного роста времени жизни носителей обусловлен изменением степени вырождения и смещением примесного рекомбинационного уровня к уровню Ферми, вызванных локальной термоупругой деформацией кристалла и соответствующим распределением концентрации неравновесных носителей заряда.

DOI: $10.21883 /$ FTP.2017.10.45010.8520

\section{1. Введение}

Как известно, кинетика фотопроводимости в кремнии определяется различными механизмами рекомбинации: рекомбинацией Шокли-Рида, межзонной рекомбинацией, безызлучательной экситонной рекомбинацией, межзонной оже-рекомбинациями. При лазерных возбуждениях проявляется нелинейная рекомбинация, обусловленная межзонной излучательной и межзонной безызлучательной оже-рекомбинацией. Известны также явления при локальном фотовозбуждении неравновесных носителей заряда (НН3), которые экспериментально и теоретически изучены и имеют физические объяснения. При низкой интенсивности собственного фотовозбуждения и исключении перезарядки рекомбинационных центров время жизни носителей не зависит от интенсивности фотовозбуждения и соответствует времени релаксации фототока. Это условие выполняется до весьма значительных плотностей фотовозбуждения кристалла, даже для скорости генерации электронно-дырочных пар равных значениям $F \approx 10^{18} \mathrm{~cm}^{-3} \cdot \mathrm{c}^{-1}[1]$.

В работах [2,3] исследован эффект аномальной релаксации фотопроводимости в кремнии при локальном, импульсном фотовозбуждении ННЗ. В этих работах обнаруженные аномалии фотопроводимости авторы работ связывают с образованием и распадом экситонов высокой плотности. В многочисленных исследованиях по рекомбинации НН3, включая и большие уровни фотовозбуждения, известных до опубликования этих работ, существенные аномалии в рекомбинации ННЗ не наблюдались. Несмотря на интерпретацию в [2,3] обнаруженной аномалии, физическое понимание явления остается недостаточным. В частности, не учтены многие характерные эффекты, возникающие при локальном интенсивном поглощении электромагнитного излучения, которые соответственно могут привести к локальному изменению физических параметров полупроводника и имеют существенное влияние на времена жизни и распределение неравновесных носителей заряда.
В данной работе исследована кинетика фотопроводимости при локальном, импульсном возбуждении собственных носителей заряда высокой концентрации в кремнии, т. е. скорости генерации электронно-дырочных пар $F \geq 10^{18} \mathrm{~cm}^{-3} \cdot \mathrm{c}^{-1}$.

Как известно, лазерное излучение с энергией кванта $\hbar \omega>E_{g}$ взаимодействует с электронной подсистемой полупроводников с последующим электрон-фононным взаимодействием и трансформацией поглощенной энергии. Поглощенное электромагнитное излучение приводит к росту температуры кристаллической решетки с возникновением в пространстве полупроводника нестационарного температурного поля, которое соответственно приводит к термоиндуцированным деформационным эффектам.

Деформация кристалла может привести к изменению времени жизни носителей заряда в полупроводниках. В работе [4] проанализировано воздействие гидростатического давления на зависимость времени жизни носителей заряда $\mathrm{Ge}$ в различных условиях, при допущении того, что эффективные массы носителей заряда и сечение захвата носителей не зависят от давления. В работе сделан вывод, что время жизни в германии $n$ и $p$ типов растет с ростом всестороннего сжатия, что объясняется приближением при давлении уровня рекомбинации к уровню Ферми. В работе [5] исследовано воздействие анизотропного давления, созданного в локальной области германиевой $p-n$-структуры, на времена жизни неосновных носителей заряда. В этой работе обнаружено, что воздействие анизотропного давления приводит к уменьшению времени жизни неосновных носителей заряда. В работе сделан вывод, что энергетическое положение рекомбинационных уровней под действием давления существенно не изменяется, а изменение времени жизни обусловлено увеличением концентрации рекомбинационных центров, генерируемых дислокациями.

В настоящем исследовании показано, что аномалии СВЧ поглощения и фотопроводимости обусловлены локальной трансформацией зонных энергетических уров- 


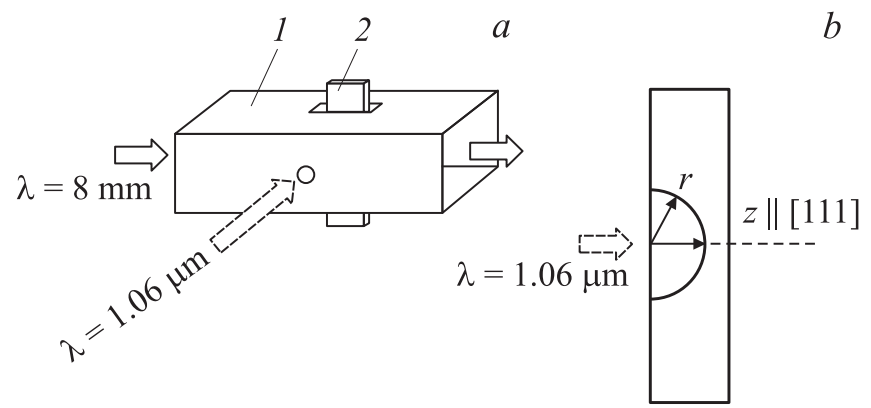

Рис. 1. Схема измерений $(a)$, геометрия и условные обозначения $(b)$ области разогрева образца. Координатными поверхностями образца являются полусферы при $r=$ const и плоскости при $z=$ const.

ней при термоупругой деформации кристалла и возникновением в образце области с повышенной концентрацией собственных носителей.

\section{2. Методика эксперимента}

Исследования проводились на линейно поляризованных волнах с частотой 35 ГГц. Образец $n$-Si с концентрацией примесей фосфора $N_{D}-N_{A}=4.0 \cdot 10^{14} \mathrm{~cm}^{-3}$, кристаллографически ориентированный вдоль направлений типа [111], размером $1.25 \times 3 \times 10$ мм помещался в волновод так, что наибольшая его поверхность была параллельна направлению вектора электрического поля СВЧ. Образец импульсно фотовозбуждался сфокусированным в пятно диаметром $\sim 0.12$ мм монохроматическим излучением с длиной волны $\lambda=1.06$ мкм, мощность $W$ которой регулировалась в диапазоне $0.01-0.5$ Вт. Сигнал модуляции СВЧ, обусловленный поглощением СВЧ поля, регистрировался по изменению прошедшей мощности на детекторе.

На рис. $1, a, b$ показаны схема измерений $(a)$, геометрия и условные обозначения $(b)$ области разогрева образца. Координатными поверхностями образца являются полусферы при $r=$ const и плоскости при $z=$ const.

\section{3. Экспериментальные результаты и их обсуждение}

На рис. 2 приведены осциллограммы поглощения СВЧ сигнала в $n$-Si при фотовозбуждении образца с интенсивностями: $A=1 \mathrm{BT} / \mathrm{cm}^{2}$ (пунктирная линия) и $A=50 \mathrm{~B} / \mathrm{cm}^{2}$ (сплошная линия), длительности импульса, равной 30 мс. При низкой и средней интенсивностях фотовозбуждения время жизни не зависит от интенсивности фотовозбуждения и равно времени релаксации фототока, осциллограммы сигналов СВЧ поглощения имеют обычную форму релаксации ФП (рис. 2, пунктирная линия). В данных исследованиях аномальная фотопроводимость проявляется при интенсивности фотовозбуждения с $A \geq 50 \mathrm{BT} / \mathrm{cm}^{2}$ и времени экспозиции $t>5.0 \mathrm{мc}$, т.е. при энергетической экспозиции более 0.25 Дж/см². При этих интенсивностях фотовозбуждения существенно изменяется форма сигнала поглощения, увеличиваются длительность и время спада сигнала, и она принимает явно аномальный вид (рис. 2, сплошная линия). Как видно, после окончания импульса фотовозбуждения сигнал СВЧ поглощения, а следовательно, и длительность неравновесной проводимости образца продолжают расти, увеличивается также длительность спада сигнала.

Как известно, при фотовозбуждении полупроводника с энергией кванта $\hbar \omega \geq E_{g}$, вследствие электронфононного взаимодействия поглощенной мощности, температура области поглощения возрастает. Так как источник тепла распределен в малом объеме, явление можно схематизировать и считать, что тепло выделяется в точечной области. Данные процессы соответствуют условиям нестационарного фотовозбуждения и нестационарного теплообмена. Изменение температуры решетки кристалла в локальной области по времени оказывается достаточно большим, но не достаточным для учета эффектов взаимодействия возникающих полей деформации и температуры. Поэтому эту задачу можно рассмотреть как квазистатическую для определенных моментов времени. Температурное поле можно определить из соответствующей нестационарной задачи теплопроводности. Предполагая, что поверхность образца теплоизолирована, распределение температуры $T(r)$ внутри от поверхности образца можно найти с помощью суперпозиции решений от точечных источников. Образованное нестационарное температурное поле имеет изотермические поверхности с концентрическими полусферами радиусом $r$. В условиях адиабатического разогрева ограниченной области полупространства, ко-

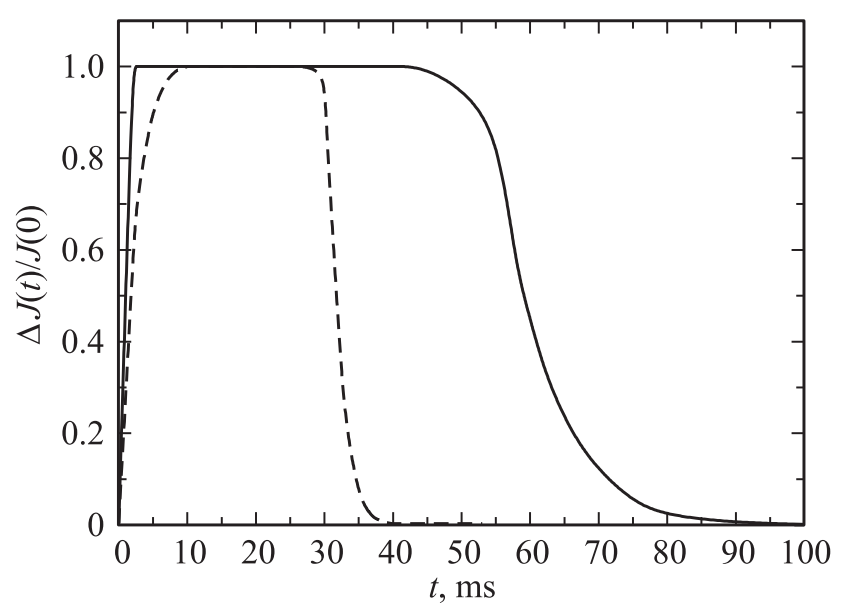

Рис. 2. Осциллограммы поглощения СВЧ сигнала в $n$-Si при фотовозбуждении образца с интенсивностями: $A=1 \mathrm{BT} / \mathrm{cm}^{2}$ (пунктирная линия) и $A=50 \mathrm{BT} / \mathrm{cm}^{2}$ (сплошная линия), $t=30 \mathrm{Mc}$. 
гда поглощаемая мощность $W \sim$ const, распределение температуры $T(r, \tau)$ по радиусу и времени определяется как [6-9]:

$$
T(r, \tau)=T_{0}+(W / 4 \pi \lambda r) \operatorname{erf} c\left(1 / 2 F_{0}^{-1 / 2}\right),
$$

где $F_{0}=\alpha \tau / r^{2}, \alpha-$ коэффициент температуропроводности, $\tau$ - время.

При непрерывном разогреве, т.е. в установившемся режиме, распределение температуры имеет более простое решение:

$$
T(r, \tau) \approx T_{0}+W / 4 \pi \lambda r
$$

После воздействия импульса фоторазогрева, в момент времени $\tau=\vartheta$, температура начинает убывать в начале координат и его окрестности, в то же время на больших расстояниях температура продолжает возрастать, что объясняется тепловой инерцией процессов как нагрева, так и охлаждения [9]. Через определенное время температура образца выравнивается и принимает свое начальное значение. Из вышеприведенного следует, что после окончания импульса разогрева распределение температуры в образце меняется по времени.

Для выяснения распределения температуры в образце в определенные моменты времени данную задачу можно представить в виде суммы решений двух задач, где результирующее температурное поле $T_{1}(r, \tau)$ будет соответствовать сумме наложения на импульс бесконечной длительности второго температурного импульса с отрицательной мощностью, который действует в той же точке $r=0$ в момент $\tau=\vartheta$. Исходя из этих значений времени можно определить и величины критериев Фурье $F(1,2)[7]$ :

$$
\begin{aligned}
T_{1}(r, \tau) & =T(r, \tau)-T(r, \tau-\vartheta)=T_{0}+(W / 4 \pi \lambda r) \\
& \times\left[\operatorname{erfc}\left(1 / 2 \cdot F_{1}^{-1 / 2}\right)-\operatorname{erfc}\left(1 / 2 \cdot F_{2}^{-1 / 2}\right)\right] .
\end{aligned}
$$

Индуцируемый поглощенной энергией локальный рост температуры вызывает тепловое расширение приповерхностных слоев, которые приводят к термоупругим напряжениям в кристалле. Координатное распределение термоупругих напряжений осесимметрично, концентрировано в малом объеме и имеет существенно неоднородный характер. В силу принципа Сен-Венана область разогрева совпадает с областью концентрации термоупругих напряжений. Температурное поле точечного поверхностного источника тепла достаточно медленно изменяется в пространстве, и тепловое расширение среды приводит к сжимающим усилиям, которые превосходят растягивающие усилия со стороны более нагретых областей. Вследствие осевой симметрии задачи, все величины зависят только от радиуса $r$ точечной области нагрева и времени $\tau$.

Для расчета термоупругих напряжений использовались соотношения теории упругости для деформаций в образце, вызванные областью разогрева без учета отвода тепла и распределения температуры по $r$ в образце, по истечении 10 мс, после воздействия импульса фоторазогрева длительностью 30 мс, на область поверхности с диаметром $\sim 0.12$ мм. Так как расстояния, на которые распространяется деформация, намного больше, чем постоянные решетки кристалла, для расчета локальных деформаций использовались те же соотношения теории упругости, что и для деформаций в полупространстве с областью разогрева в квазистатической постановке.

Радиальные и тангенциальные компоненты термоупругих напряжений, в период нагрева $0<r<\vartheta$, можно определить по следующим формулам:

$$
\begin{aligned}
& \sigma_{r r}=[2 G /(1-\mu)]\left[\varepsilon_{r r}+\mu \varepsilon_{\varphi \varphi}-(1+\mu) \alpha_{r} T_{r}\right], \\
& \sigma_{\varphi \varphi}=[2 G /(1-\mu)]\left[\varepsilon_{\varphi \varphi}+\mu \varepsilon_{r r}-(1+\mu) \alpha_{r} T_{r}\right],
\end{aligned}
$$

где $\varepsilon_{r r}=\partial u / \partial r, \varepsilon_{\varphi \varphi}=u / r, u$ - радиальное перемещение, $\sigma_{r r}$ - радиальные термоупругие напряжения, $\sigma_{\varphi \varphi}-$ тангенциальные термоупругие напряжения в радиальных слоях, $G=E / 2(1+v)$ - модуль сдвига, $E$ - модуль упругости, $\mu-$ коэффициент Пуассона, $\alpha_{r}$ - коэффициент теплового расширения по изотермическому радиусу $r, T(r)$ - распределение температуры по радиусу $r$, остальные обозначения показаны на рис. 1 .

Учет температурных и кристаллографических зависимостей механических параметров: модуля Юнга $E$, коэффициента Пуассона $\mu$ представляет значительные трудности, поэтому для вычислений использовались следующие постоянные значения: $E_{[111]}=190 \cdot 10^{3} \mathrm{MPa}, E_{[100]}=$ $=130 \cdot 10^{3} \mathrm{MPa}, \mu=0.3$, а коэффициент линейного теплового расширения $\alpha=\left(2.6+0.0025 \Delta T \mathrm{~K}^{-1}\right) \cdot 10^{-6} \mathrm{~K}^{-1}$.

Из анализа проявляющихся компонентов термоупругих напряжений в кристалле наиболее существенными являются сдвиговые напряжения в радиальных слоях полусферического объема фотовозбужденной части образца [10-12].

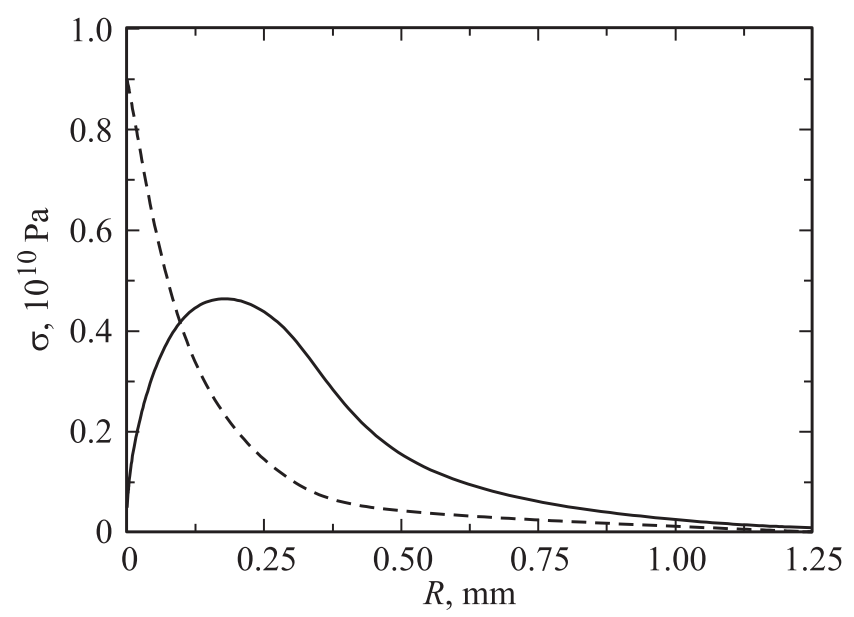

Рис. 3. Распределение сдвиговых термоупругих напряжений по $r$ (пунктирная линия) при воздействии импульса фотовозбуждения и по истечении времени 10 мс после окончания импульса фотовозбуждения (сплошная линия). 


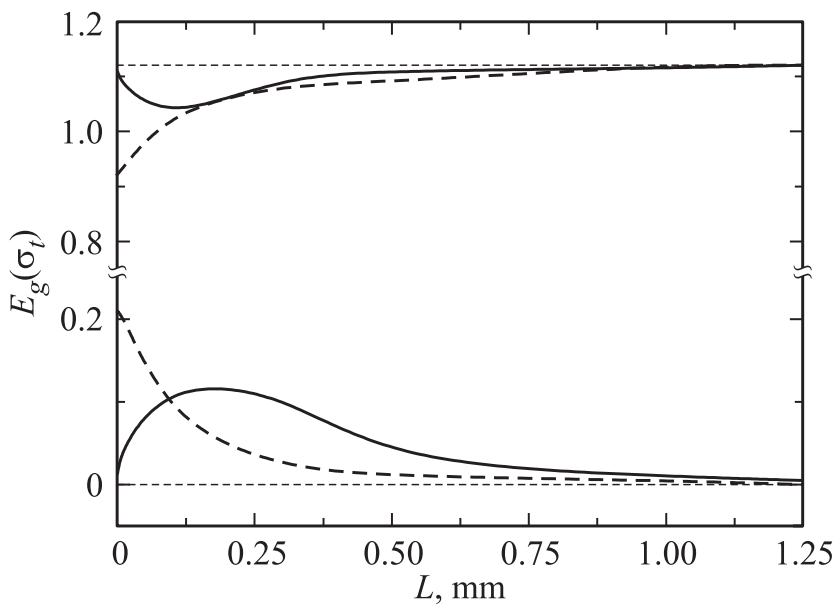

Рис. 4. Зависимости смещений энергетических уровней $E_{c}$ и $E_{v}$, обусловленные термоупругой деформацией кристалла в направлении $r \|[111] . E_{c}$ и $E_{v}$ после воздействия импульса фотовозбуждения (пунктирная линия) и по истечении 10 мс, после окончания импульса фотовозбуждения (сплошная линия).

Распределение радиальных сдвиговых термоупругих напряжений $\sigma_{\varphi \varphi}$ с учетом граничных условий можно рассчитать по следующей формуле [11]:

$$
\sigma_{\varphi \varphi}=-\sigma_{r r}-E \alpha T(r) .
$$

Распределение сдвиговых термоупругих напряжений по $r$ (пунктирная линия) при воздействии импульса фотовозбуждения и по истечении времени 10 мс после окончания импульса фотовозбуждения (сплошная линия) показаны на рис. 3.

Зависимости смещений энергетических уровней $E_{c}$ и $E_{v}$, обусловленные термоупругой деформацией кристалла в направлении $r \|$ [111], показаны на рис. 4.

Сжатие кремния по направлениям типа [111] и [100] приводит к уменьшению ширины запрещенной зоны вследствие смещения долин зоны проводимости и расщепления уровней валентной зоны $[13,14]$. Данная асимметрично-градиентная упругая деформация с радиальными изменениями параметров зонной структуры и радиальным градиентом плотности состояний приводит к перераспределению концентрации носителей заряда по долинам в направлении координаты $r$, так как деформация снимает вырождение дна зоны проводимости и соответственно вырождение примесного уровня. При этом расщепление примесных уровней приближенно совпадает с расщеплением дна зоны, так как энергия ионизации примеси слабо зависит от давления.

С уменьшением ширины запрещенной зоны на $\Delta E_{g}$ увеличивается концентрация собственных носителей заряда:

$$
n_{i}=n_{i 0} \exp \left(-\Delta E_{g} / 2 k T\right) .
$$

Смещение примесных уровней под действием деформации и изменение степени их вырождения приводит к влиянию давления на процесс генерации-рекомбинации носителей.
Скорость изменения $\tau_{i}$ при деформации определяется выражением [14]

$$
d \tau_{i} / d_{\varepsilon}=\left[\left(\tau_{p 0} n_{i}+\tau_{n 0} p_{i}\right) / 2 k T n_{i}\right](d \chi / d \varepsilon)
$$

где $\chi=E_{t}-\left(E_{v}+E_{g} / 2\right)-$ расстояние уровня ловушки от середины запрещенной зоны.

Как следует из выражения (8), изменение $d \tau_{i} / d \varepsilon$ определяется скоростью изменения расстояния между уровнем ловушки $E_{t}$ и уровнем Ферми $E_{\mathrm{F}}$. При этом зависимость времени жизни от деформации тем больше, чем меньше степень легирования, т.е. в полупроводниках, близких к собственным.

\section{4. Заключение}

Таким образом, локальное импульсное фотовозбуждение собственных неравновесных носителей заряда высокой концентрации в кремнии приводит к увеличению температуры в ограниченной области образца, приводящей к локальной термоупругой деформации кристалла. Данная деформация изменяет ширину запрещенной зоны и соответственно энергию и степень вырождения рекомбинационных уровней относительно уровня Ферми, что приводит к изменению их заселенности и к увеличению времени жизни неравновесных носителей тока.

\section{Список литературы}

[1] Г.И. Галкин. Тр. ФИАН, 128, 3 (1981).

[2] П.А. Бородовский, А.Ф. Булдыгин, А.С. Токарев. Микроэлектроника, 35 (6), 403 (2006).

[3] П.А. Бородовский, А.Ф. Булдыгин, С.В. Голод. ФТП, 43 (3), 329 (2009).

[4] W.J. Eresain, J.R. McKeivey. J. Appl. Phys., 41 (12), 4963 (1970).

[5] В.И. Гаман, В.Ф. Агафонников. Изв. вузов. Сер. Физика, 12, 97 (1967).

[6] Г. Каслоу, Д. Егер. Теплопроводность твердых тел (М., Наука, 1964) с. 238. [Пер. с англ.: H.S. Carslaw, J.C. Jaeger. Conduction of heat in solids (Oxford, 1959)].

[7] А.И. Пехович, В.М. Жидких. Расчеты теплового режима твердых тел (Л., Энергия, 1976) ч. 1, с. 70; ч. 2, c. 316 .

[8] Б.А. Григорьев. Импульсный нагрев излучениями (М., Наука, 1974) ч. 1 , с. 261 ; ч. 2, с. 213.

[9] Ф.М. Камья. Импульсная теория теплопроводности (М., Энергия, 1972) с. 52. [Пер. с англ.: F.M. Camia. Traite de thermocinetique impulsionneelle par (Paris, 1967)].

[10] Э. Мелан, Г. Паркус. Термоупругие напряжсения вызываемые стационарными температурными полями (М., Гос. изд-во физ.-мат. лит., 1958) с. 111. [Пер. с англ.: E. Melan, H. Parkus. Warmespannungen infolge stationarer temperaturfelder (Wien, Springer Verlag, 1953)].

[11] Г. Паркус. Неустановивщиеся температурные напряж⿻ения (М., Гос. изд-во физ.-мат. лит., 1963) с. 100. [Пер. с англ.: H. Parkus. Instationare warmespannungen (Wien, Springer Verlag, 1959)]. 
[12] Б. Боли, Дж. Уэйнер. Теория температурных напряжений (М., Мир, 1964) с. 165, 259. [Пер. с англ.: В.В. Boley, J.H. Weiner. Theory of thermal stresses (N.Y.-London, 1960)].

[13] Г.Л. Бир, Г.Е. Пикус. Симметрия и деформационные эфбекты в полупроводниках (М., Наука, 1972) с. 374.

[14] А.Л. Полякова. Дебормащия полупроводников и полупроводниковых приборов (М., Энергия, 1979) с. 38.

Редактор Г.А. Оганесян

\title{
Effects of the local photo-excitation of high concentration carriers in silicon
}

\author{
A.M. Musaev \\ Institute of Physics, Daghestan Scientific Center, \\ Russian Academy of Sciences, \\ 367003 Makhachkala, Russia
}

Abstract The phenomena at the local impulse photo-excitation of intrinsic high concentrated non-equilibrium carriers are experimentally studied. The effect of substantial increase in photoexcited carrier lifetime is found. It is shown that the effect of substantial increase in photoexcited carrier lifetime is stemmed from a change in a degeneration level and the displacement of an impurity recombination level towards the Fermi level caused by the local thermoelastic defomation of the crystal and corresponding distribution of non-equilibrium carrier concentration. 